ISSN-PRINT

1794-9831

E-ISSN 2322-7028

Vol. 14 No. 2

Jul - Dic 2017

Cúcuta, Colombia

Recibido:

5 de Septiembre de 2016

Aprobado:

15 Mayo de 2017

* Enfermera. Correo electrónico: lore-92-bon@ hotmail.com. Orcid: 00000002-3375-9043.

Fundación Hospital San Carlos.

Bogotá, Colombia.

** Enfermera.

Magister en

Docencia

Universitaria,

Énfasis Educación.

Correo: rcoral@

udca.edu.co.

Orcid 0000-

0002-0244-5578.

Docente programa de enfermería.

Universidad de

Ciencias Aplicadas

y Ambientales

U.D.C.A. Bogotá

D.C., Colombia.

*** Enfermera

Magister en

Enfermería con

énfasis en Gerencia

de Servicios de

Salud. Correo:

luvargas@udca.

edu.co. Orcid:

0000-0002-7674-

9881 Docente

programa de

enfermería.

Universidad de

Ciencias Aplicadas

y Ambientales

U.D.C.A. Bogotá

D.C., Colombia.

\section{Perfil sociodemográfico, condiciones de salud y atención a personas con diabetes}

\section{Socio-demographic profile, health conditions and care for people with diabetes.}

\section{Perfil sócio demográfico, condições de saúde e cuidados para as pessoas com} diabetes

\author{
Meiby Lorena Rojas-Castañeda* \\ Rosa del Carmen Coral-Ibarra** \\ Luz Dalila Vargas-Cruz***
}

\section{Para citar este artículo / To reference this article / Para citar este artigo/}

Rojas-Castañeda ML, Coral-Ibarra RC, Vargas-Cruz LD. Perfil sociodemográfico, condiciones de salud y atención a personas con diabetes. Rev. cienc. cuidad. 2017;14(2):22-37

\section{Resumen}

Objetivo: Analizar el perfil sociodemográfico, condiciones de salud y atención a personas con diabetes, a partir de la revisión de historias clínicas. Materiales y Métodos: Estudio descriptivo transversal. La población fue de 821 historias clínicas de pacientes con diabetes mellitus. En total la muestra fue de 230 historias clínicas por método aleatorio simple. Se utilizó el instrumento Impacto en la Condición de Salud y Calidad de Vida de las Atenciones de Salud en Pacientes Diabéticos Tipo 2 de Macul, que evalúa el perfil sociodemográfico, las condiciones de salud y el tipo de atención otorgada. Resultados: $39 \%$ de los pacientes presentó el valor de la última hemoglobina glicosilada mayor de $12 \%$, el 41,3\% no presentó ningún riesgo de pie diabético, 46,9\% tuvo diagnóstico de obesidad y el $23,9 \%$ presentó sobrepeso. El porcentaje de cumplimiento de citas al mes por parte del médico y la enfermera fue del $100 \%$, a diferencia del control por parte de la nutricionista que debe realizase cada 6 meses, sólo se registró un cumplimiento del $71,7 \%$. No se encontró relación estadísticamente significativa entre asistencia al programa con adherencia al tratamiento, o con compensación de la enfermedad. Conclusiones: Los pacientes con diabetes a pesar de estar en un programa de atención integral, no tienen un óptimo control glicémico, lo cual sugiere la revisión del cumplimiento del régimen terapéutico por parte de los profesionales de la salud para prevenir complicaciones que se puedan presentar a corto, mediano o largo plazo.

Palabras clave: Atención a la salud, complicaciones crónicas, diabetes mellitus tipo 2, enfermedad crónica.

\section{Abstract}

Objective: To analyze the socio-demographic profile, health conditions and attention to people with diabetes from a review of medical records. Materials and Methods: Cross-sectional descriptive study. The population was of 821 medical records of patients with diabetes mellitus. In total the sample was of 230 medical records through a simple aleatory method. The Impact instrument was used in the Health Condition and Life Quality of the Health Care in Diabetic Patients Type 2 from Macul, that evaluates the socio-demographic profile, the health conditions and the type of attention given. Results: $39 \%$ of the patients presented the value of the last glycosylated hemoglobin greater than $12 \%, 41,3 \%$ did not present any risks of diabetic foot, $46,9 \%$ had an obesity diagnose and $23,9 \%$ presented overweight. The doctor and the nurse percentage of compliance of appointments per month was $100 \%$, as opposed to the control by the nutritionist that must be performed every 6 months, only a of $71,1 \%$ of compliance was registered. A statistically significant relationship was not found between compliance to the program and treatment adherence, or with compensation for 
Conclusions: Patients with diabetes despite of being in a program of comprehensive care, do not have an optimal glycemic control, which suggests a review of the compliance of the therapeutic regime by the health professionals to prevent complications that may occur in a short, medium, or long term.

Keywords: Health care, chronic complications, diabetes mellitus type 2, chronic disease.

ISSN-PRINT

$1794-9831$

E-ISSN 2322-7028

Vol. 14 No. 2

Jul - Dic 2017

Cúcuta, Colombia

\section{Resumo}

Objetivo: Analisar o perfil sócio demográfico, condições de saúde e cuidados para as pessoas com diabetes a partir da revisão de histórias clínicas. Materiais e Métodos: Estudo descritivo transversal. A população foi de 821 historias clínicas de pacientes com diabetes mellitus. Em total a amostra foi de 230 historias clínicas por método aleatório simples. Utilizou-se o instrumento Impacto na Condição de Saúde e Qualidade de Vida das Assistências Médicas em Pacientes Diabéticos Tipo 2 de Macul, que avalia o perfil sócio demográfico, as condições de saúde e o tipo de atendimento recebido. Resultados: $39 \%$ dos pacientes apresentaram o valor da última hemoglobina glicada ( $\mathrm{Hb} \mathrm{A} 1 \mathrm{c}$ ) maior de $12 \%$, o 41,3\% não apresentou nenhum risco de pé diabético, 46,9\% tiveram diagnóstico de obesidade e o 23,9\% apresentaram excesso de peso. A porcentagem de cumprimento de consultas ao mês por parte do médico e a enfermeira foi do $100 \%$, a diferença do controle por parte da nutricionista que deve realizar-se a cada 6 meses, apenas registrou-se um cumprimento do $71,7 \%$. Não se encontrou relação estatisticamente significativa entre assistência ao programa com aderência ao tratamento, ou com compensação da doença. Conclusões: Os pacientes com diabetes a pesar de estar num programa de atendimento integral, não tem um óptimo controle glicémico, o qual sugere a revisão do cumprimento do regime terapêutico por parte dos professionais da saúde para prevenir complicações que se possam apresentar a curto, médio ou longo prazo.

Palavras-chave: Assistência médica, complicações crónicas, diabetes mellitus tipo 2, doença crónica.

\section{Introducción}

La diabetes mellitus tipo 2 (DM 2) representa un problema de salud pública y se ha considerado como una verdadera epidemia debido a su alta prevalencia (1). En el mundo hay más de 347 millones de personas con esta enfermedad; más del $80 \%$ de las muertes se registran en países de ingresos bajos y medios; casi la mitad de esas muertes corresponde a personas de menos de 70 años y un $55 \%$ son mujeres. La Organización Mundial de la Salud (OMS) afirmó que las muertes por DM Tipo 2 se multiplicarán por dos entre los años 2005 al 2030 (2).

Entre el $50 \%$ y el $80 \%$ de las muertes de pacientes diabéticos se debe a causas cardiovasculares, aunque también influye la falta de acceso a los servicios de salud y a los medicamentos esenciales, lo cual puede producir complicaciones como ceguera, insuficiencia renal y la necesidad de realizar amputación de extremidades. Otras condiciones que complican a los pacientes son el sobrepeso, la obesidad y la inactividad física (3).
En Colombia, en el año 2012, murieron 7.154 personas por esta enfermedad, con una tasa de mortalidad de 15,36 muertes por cada 100.000 habitantes. En el año 2010, el porcentaje de cobertura de pacientes diabéticos controlados entre los 18 y los 29 años fue de $9,82 \%$ y en las edades de 50 a 69 años fue del $30 \%$. Estas cifras son preocupantes si se tiene en cuenta que la DM Tipo 2 no controlada conlleva a complicaciones graves (4). En Bogotá, la DM Tipo 2 es una de las primeras causas de morbilidad, además de la hipertensión y la enfermedad coronaria; específicamente en la localidad de Bosa, ocupa el tercer lugar entre las patologías mencionadas, con un $22 \%(5)$.

Abordar el problema de las enfermedades crónicas es una situación compleja que lleva consigo aceptar la enfermedad y el régimen terapéutico durante toda la vida. La representación social de esta enfermedad se asocia negativamente con muerte, discapacidad y daños en el organismo. Desde el punto de vista económico, todavía existe una población socialmente desfavorecida, con escaso o nulo acceso a los servicios 
ISSN-PRINT

1794-9831

E-ISSN 2322-7028

Vol. 14 No. 2

Jul - Dic 2017

Cúcuta, Colombia básicos y a la atención de la salud (5). "En Colombia, el incremento de las enfermedades crónicas no trasmisibles, se vive en medio de un Sistema General de Seguridad Social en Salud (SGSSS) frágil, con ausencia de propuestas concretas para el manejo integral de estas personas y sus familias" (6).

Uno de los impactos que más se genera a causa de la DM Tipo 2 está relacionado con los altos costos. "En el caso de América Latina y el Caribe (Argentina, Brasil, Colombia y México), la pérdida de la productividad económica asociada a las enfermedades crónicas, entre el 2006 y el 2015, podría resultar en US $\$ 84$ billones y de US\$13,54 billones para la enfermedad cardiovascular y diabetes" (7). La carga económica está representada en los "costos directos (medicamentos, hospitalización, consultas, manejo de complicaciones) e indirectos de la enfermedad (el abordaje de capital humano debido a la muerte prematura y la discapacidad)" (6). Todos estos costos van acompañados del tratamiento que se le indica al paciente; al respecto, se ha reportado que el uso de hipoglucemiantes orales es de mayor efectividad y menor costo en comparación con los esquemas que incluyen insulina e hipoglucemiantes orales (8).

Esta enfermedad "genera profundos impactos relacionados con las complicaciones a corto plazo (hipoglucemia) y a largo plazo (enfermedad cardiovascular, neuropatía, nefropatía y retinopatía) por lo que afecta tanto la duración como la calidad de la vida" (9). "Lo anterior conduce a la necesidad de mejorar los procesos de atención que se ofrecen a los pacientes" (10), teniendo en cuenta que "los programas de control representan una valiosa herramienta para la inclusión de prácticas saludables" (10).

Estos programas se pueden implementar, pero el paciente debe estar comprometido con el cuidado de su enfermedad (11). Las acciones deben estar dirigidas a retardar su progresión y prevenir las complicaciones agudas y crónicas (12). Esto implica que las instituciones prestadoras de salud deben definir las conductas de seguimiento y control del paciente, en donde se incluya la valoración por especialidades médicas (13).

En Colombia, el programa de control de la diabetes debe desarrollarse en todas las instituciones del país, de acuerdo con las normas del Sistema General de Seguridad Social en Salud (SGSSS) fijadas por la Ley
100 de 1993 y la aplicación de la Guía de práctica clínica para el diagnóstico, tratamiento y seguimiento de la DM Tipo 2 de 2015 (14-55). "Para optimizar la adherencia, es recomendable establecer mecanismos eficientes de seguimiento y de rescate de los pacientes que no cumplan con los controles establecidos" (15) y, por ello, se ha incluido como estrategia el llamado telefónico al paciente para el manejo de los medicamentos en los horarios prescritos (16) y se destaca la educación a estos pacientes; sin embargo, sólo un reducido número de ellos, en algunos países, recibe una adecuada educación diabetológica (17).

Esta situación suscita el interés por analizar el perfil sociodemográfico de la población de un programa de atención a pacientes con diabetes mellitus tipo 2, para identificar los factores sociodemográficos, la condición de salud y el tipo de atención otorgada; así mismo, para conocer las necesidades de mejoramiento del programa, lo cual es de gran interés para los profesionales de salud que trabajan en él y la institución participante. El periodo de ejecución del proyecto fue de un 1 año y medio.

\section{Materiales y métodos}

La investigación corresponde a un estudio descriptivo transversal que permite dar cuenta de los fenómenos, situaciones, contextos y sucesos, especificando las propiedades, características y perfiles de grupos y de comunidades. La población fue de 821 historias clínicas de pacientes con diabetes tipo 1 y 2 que recibieron atención entre el periodo de julio del 2014 a julio del 2015, obtenidas a partir de la base de datos manejada por el programa de diabetes de la institución. Se escogieron 701 historias clínicas de acuerdo con los criterios de inclusión y exclusión planteados para el estudio.

De este total se seleccionó una muestra de 230 historias clínicas por el método aleatorio simple, con un 95 $\%$ de confiabilidad y un $5 \%$ al error de estimación, utilizando la fórmula de muestreo probabilístico y conociendo el tamaño de la población.

Los criterios de inclusión fueron: pacientes con edad igual o mayor a 30 años e inscritos en el programa de diabetes. Los criterios de exclusión fueron: inasistencia al programa por un periodo mayor a 2 meses y pacientes con diagnóstico de diabetes mellitus tipo 1. 
La recolección de la información se realizó a través del archivo de historias clínicas de la institución participante, mediante la utilización de un instrumento denominado Impacto en la Condición de Salud y Calidad de Vida de las Atenciones en Salud en Pacientes Diabéticos Tipo 2 de Macul (18). Dicho instrumento fue validado por expertos y por medio de una prueba piloto, autorizado por vía electrónica, y entre sus categorías se encuentran factores sociodemográficos, condición de salud y tipo de atención otorgada, para un total de 31 ítems, clasificados en preguntas cerradas -en su mayoría de tipo escala Likert, unas con elección única dicotómicas y politómicas, y otras pocas consideradas como preguntas mixtas, dando la opción de respuesta abierta (otro)-. La confiabilidad y validez del instrumento fueron probadas con un grupo de estudio pequeño de 30 personas, un mes antes de la aplicación del mismo, encontrando respuestas a las variables planteadas.

La técnica de análisis de datos y sistematización de la información se realizó a través del programa de Excel de 2010, con tablas dinámicas.

Elproyecto fue aprobado por la institución participante, por la dirección del programa de diabetes de la misma institución -mediante una carta de autorización-, por el comité de ética de la universidad a través de la presentación de la ficha educativa y la aprobación de la misma por parte de los miembros de la junta, y por los pacientes mediante un consentimiento informado debidamente firmado. La clasificación del estudio es de riesgo mínimo, según la Resolución No. 8430 de 1993 del Ministerio de Salud (19) y se tuvo en cuenta, además, la declaración de Helsinki y lo dispuesto por el Consejo de Organizaciones de las Ciencias Médicas (CIOMS) relacionado con las pautas éticas internacionales para la investigación biomédica en seres humanos (20).

\section{Objetivos}

\section{Objetivo general}

Analizar el perfil sociodemográfico, condiciones de salud y atención a personas con diabetes tipo II a partir de la revisión de historias clínicas.

\section{Objetivos específicos}

- Describir los factores sociodemográficos incluyendo el tiempo de enfermedad y adherencia en el tratamiento.
- Determinar la condición de salud, el diagnóstico médico de patología crónica agregada, las complicaciones y la compensación de la enfermedad.

- Identificar el tipo de atención otorgada por parte de los profesionales de la salud a la población estudiada.

\section{Resultados}

Durante el periodo de recolección de información no se presentaron limitaciones. Los resultados de la caracterización se presentan de acuerdo con los siguientes aspectos: factores sociodemográficos, condición de salud y tipo de atención otorgada en un programa de atención integral de diabetes.

\section{Factores sociodemográficos}

En relación con los factores sociodemográficos, los porcentajes más altos del estado civil de los pacientes correspondieron a solteros y casados, con 29,5 \% y 23,9\% respectivamente. De igual manera, el número de pacientes viudos se muestra significativo con un porcentaje de 19,5\%.

El mayor porcentaje de pacientes con diabetes mellitus tipo 2 se encontró entre las edades de 50 a 59 años (30 $\%)$ y, en igual porcentaje, entre las edades de 60 a 69. El $73 \%$ de los pacientes pertenece al género femenino, mientras el $27 \%$ hace parte del género masculino.

En la variable ocupación se observa que el $91.3 \%$ de pacientes se dedica al hogar y el restante trabaja en oficios varios. Se encontró que las mujeres dedicadas al hogar o jubiladas asisten con mayor frecuencia a las instituciones de salud, lo cual puede ser benéfico para el control de su enfermedad y la prevención de complicaciones. En este estudio, sólo el 5,63 \% reportó no adherencia al tratamiento, correspondiente a personas de sexo femenino y con edad mayor a 60 años.

En relación con el tiempo, en años, que llevan las personas con el diagnóstico de la enfermedad, el 6,5 $\%$ de los pacientes llevaba menos de un año, el 28,2 $\%$ de 1 a 5 años, el $26 \%$ de 6 a 10 años y el mayor porcentaje correspondió a más de 10 años, con un $39 \%$. También para este grupo se encontró la mayor adherencia al tratamiento, con un 41,4 \% (ver Figura 1). La correlación evidencia relación estadísticamente significativa $(\mathrm{P}<0,05)$ entre el tiempo de la enfermedad y la adherencia al tratamiento. 
ISSN-PRINT

1794-9831

E-ISSN 2322-7028

Vol. 14 No. 2

Jul - Dic 2017

Cúcuta, Colombia

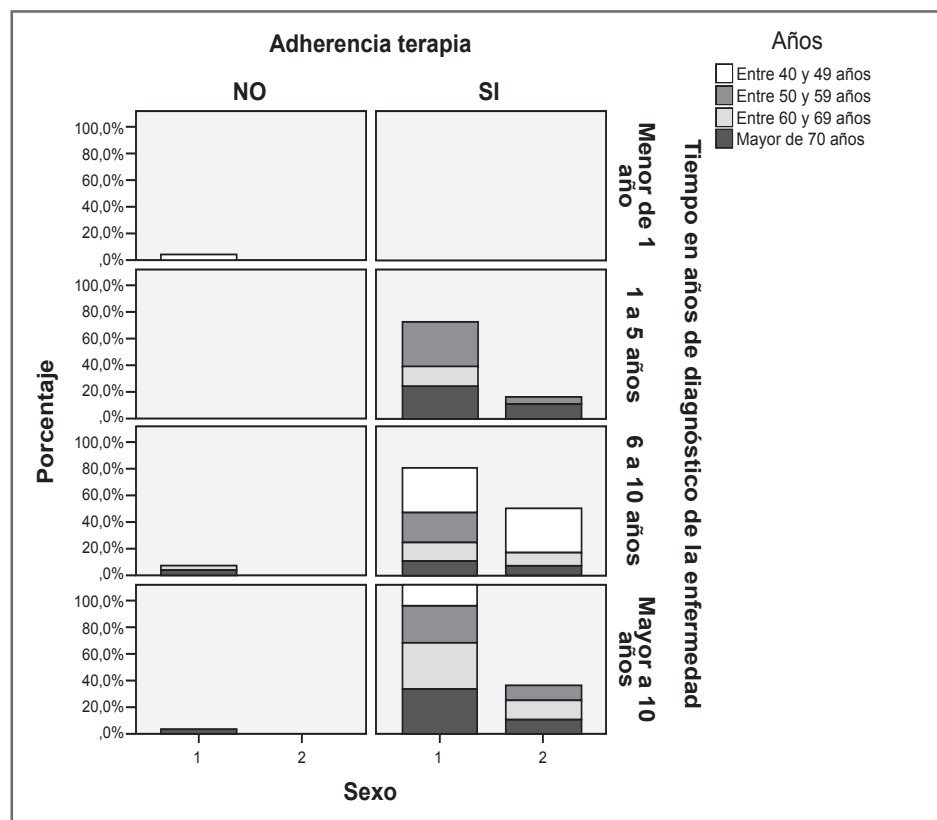

Figura 1. Adherencia al tratamiento.

Fuente: Impacto en la condición de salud y calidad de vida de las atenciones de salud en pacientes diabéticos tipo 2 de Macul, 2015.
El 69,5\% equivale a los pacientes que llevan menos de un año en el programa y el 30,4\% hace referencia a los pacientes que llevaban de 1 a 5 años.

\section{Condición de salud}

En relación con la condición de salud de los pacientes, específicamente sobre los resultados de hemoglobina glicosilada ( $\mathrm{HbAlc})$, en los últimos tres meses únicamente el $21 \%$ tiene cifras dentro de los parámetros normales, considerado como valor normal menor a $7 \%$ (21). Este examen permite evaluar los factores relacionados con la adherencia del paciente y la calidad de su control por el equipo de salud y posibilita, además, identificar el riesgo de desarrollar complicaciones microvasculares y evaluar la necesidad de ajustar su tratamiento (22). Al relacionar estos datos con el nivel de compensación se encontró que un $72,2 \%$ de los pacientes de este estudio están descompensados.
Se realizó prueba de chi-cuadrado para relacionar compensación de la enfermedad con tiempo en años de diagnóstico de la enfermedad $(0,74)$, con tiempo en años de control en el programa $(0,76)$ y con adherencia al tratamiento $(0,2)$. Estos resultados, si resultan mayores a 0,05 , evidencian que no hay relación estadísticamente significativa entre las variables.

Con respecto al diagnóstico médico de patología crónica agregada, el 80,9 \% presenta hipertensión arterial, el 17,3 \% tenía hipotiroidismo y, en menores porcentajes, se presentó artritis reumatoide, osteoporosis, esquizofrenia, enfermedades cardiovasculares, Enfermedad Pulmonar Obstructiva Crónica (EPOC), tuberculosis y cáncer, entre otras. La Figura 2 muestra que el mayor porcentaje de complicación por la diabetes fue la nefropatía diabética $(72,6 \%)$. 


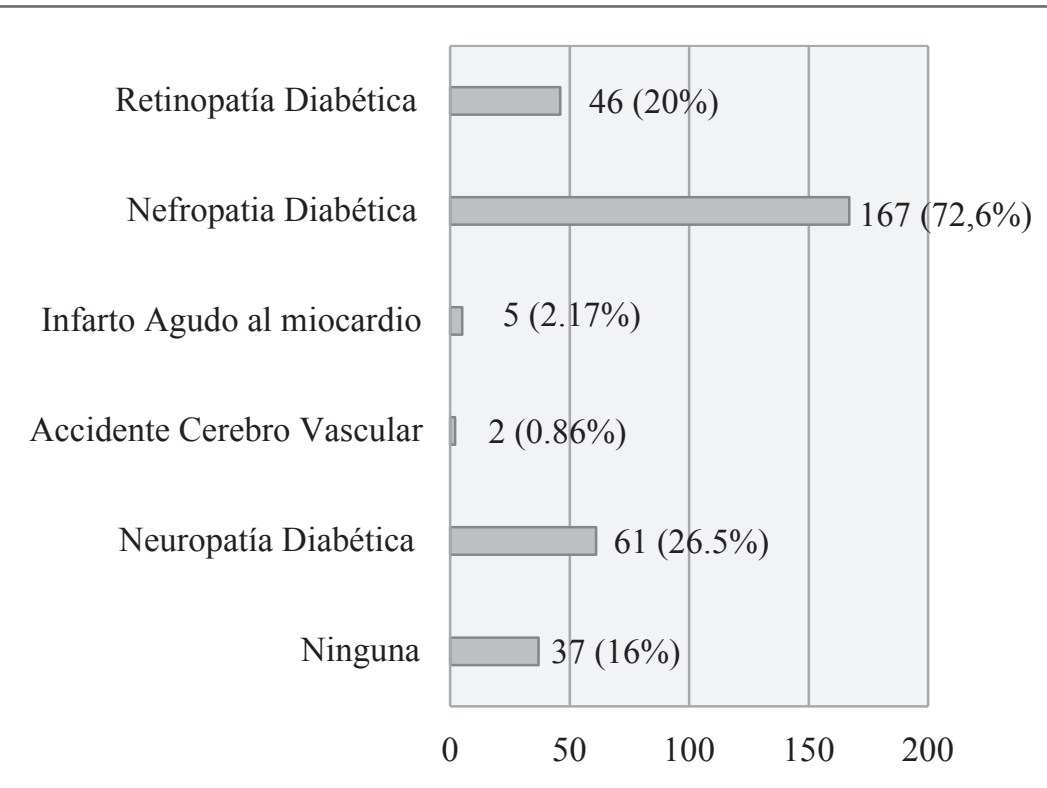

ISSN-PRINT

1794-9831

E-ISSN 2322-7028

Vol. 14 No. 2

Jul - Dic 2017

Cúcuta, Colombia

Figura 2. Diagnóstico médico de complicaciones de la diabetes.

Fuente: Impacto en la condición de salud y calidad de vida de las atenciones de salud en pacientes diabéticos tipo 2 de Macul. 2015.

El 41,3\% de los pacientes no tiene riesgo de pie diabético; sin embargo, en un alto porcentaje no hay registro de la información en la historia clínica $(55,7$ $\%)$.

El 46,9\% de los pacientes se encuentra con diagnóstico de obesidad y el 23,9 \% con sobrepeso, situación que pone en riesgo a estos pacientes de desarrollar enfermedad cardiovascular (23). De acuerdo con la Figura 3, se encontró diferencia entre las personas compensadas y descompensadas para cada grupo; $\sin$ embargo, la significancia es de 0,20 , comprobando que no hay relación estadísticamente significativa entre los pacientes que presentan obesidad y su estado de compensación o no compensación de la enfermedad.

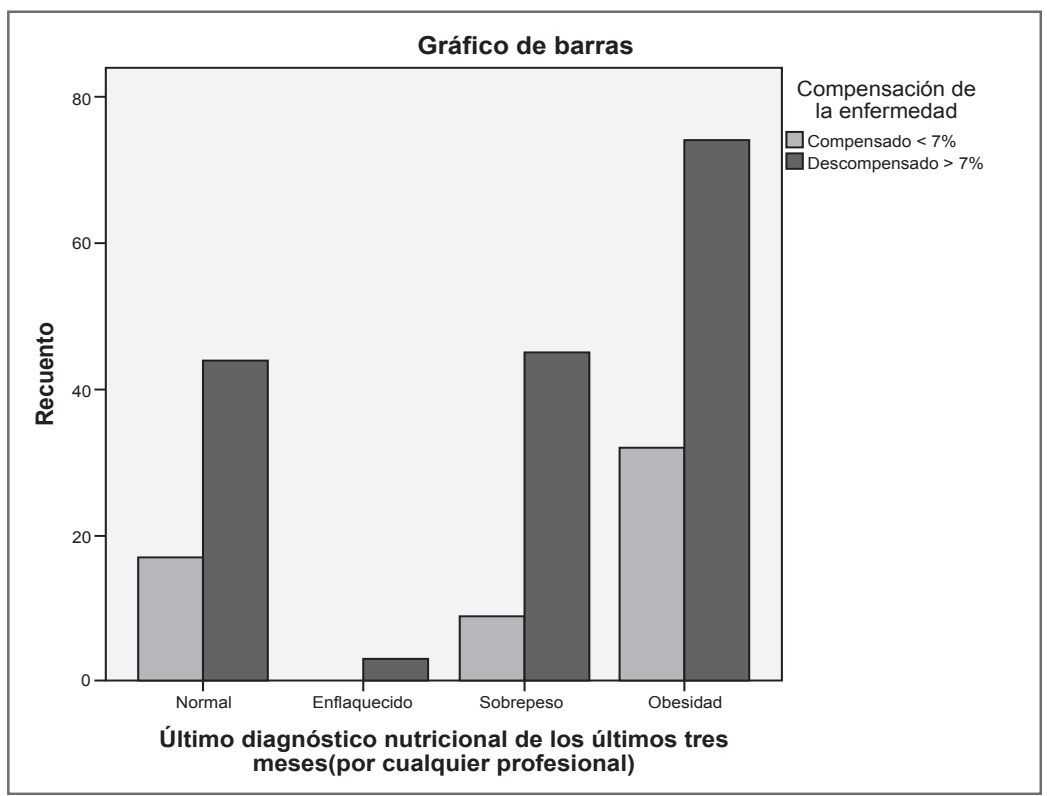

Figura 3. Último diagnóstico nutricional de los últimos tres meses.

Fuente: Impacto en la condición de salud y calidad de vida de las atenciones de salud en pacientes diabéticos tipo 2 de Macul. 2015 
ISSN-PRINT

1794-9831

E-ISSN 2322-7028

Vol. 14 No. 2

Jul - Dic 2017

Cúcuta, Colombia

\section{Tipo de atención otorgada}

En relación con la variable tipo de atención otorgada, el porcentaje de cumplimiento de citas al mes por parte del médico y la enfermera fue del $100 \%$. El control de la nutricionista, que es semestral, correspondió al 71,7 $\%$, y del ortopedista, que se debe realizar cada año, fue del $51 \%$. Estadísticamente no se evidenció diferencia significativa entre las variables de estado nutricional y el control por los profesionales de la salud.

Tabla 1. Examenes de laboratorio y pruebas diagnósticas solicitadas en el último año.

\begin{tabular}{llll}
\hline \multicolumn{1}{c}{ Exámenes } & Solicitados & Realizados & $\begin{array}{c}\text { Porcentaje de } \\
\text { cumplimiento (\%) }\end{array}$ \\
\hline Glicemia & 230 & 219 & $95,2 \%$ \\
\hline $\begin{array}{l}\text { Hemoglobina } \\
\text { Glicosilada }\end{array}$ & 230 & 219 & $95,2 \%$ \\
\hline Perfil lipídico & 201 & 198 & $98,5 \%$ \\
\hline Creatinemia & 182 & 181 & $99,4 \%$ \\
\hline Orina Completa & 174 & 173 & $99,4 \%$ \\
\hline Microalbuminuria & 167 & 167 & $100 \%$ \\
\hline Electrocardiograma & 46 & 17 & $37 \%$ \\
\hline Fondo de ojo & 141 & 103 & $73 \%$
\end{tabular}

Fuente: Impacto en la condición de salud y calidad de vida de las atenciones de salud en pacientes diabéticos tipo 2 de Macul. 2015

Diferentes exámenes de control se realizaron a los pacientes asistentes al programa durante el año inmediatamente anterior al presente estudio. A los pacientes se les solicitó y se les realizó la HbAlc y la glicemia cada tres meses, con un porcentaje alto de cumplimiento como se observa en la Tabla 1. De igual manera, se solicitaron y se realizaron otros exámenes con alto cumplimiento, excepto el electrocardiograma con un porcentaje del $37 \%$. Se encontró que el tipo de tratamiento hipoglicemiante que con mayor frecuencia se prescribe es el mixto (65 $\%)$, seguido por el de vía oral (27 \%) e insulina (8 \%).

En relación con la educación o instrucción brindada a los pacientes sobre el régimen alimenticio, se halló que el $65,2 \%$ tiene registro de la educación ofrecida por el profesional en nutrición.

Al respecto del registro de educación por parte del profesional de enfermería, correspondió a un cumplimiento del $100 \%$ de los pacientes en las variables de alimentación, actividad física y su enfermedad, excepto en cuidado de los pies. Las actividades de educación que se registraron por parte del profesional de enfermería en el programa fueron a través de talleres educativos sobre estilos de vida saludables, complicaciones crónicas de la diabetes, insulinas y automonitoreo.

En el caso del registro de la educación por parte del médico fue igual que el de la (el) enfermera(o), excepto el registro sobre el cuidado de los pies; sin embargo, se evidencia que se refieren los pacientes a ortopedia y, específicamente, a la especialidad de podología en un $86,9 \%$ de los pacientes, una vez al año.

\section{Discusión}

la diabetes mellitus tipo 2 constituye uno de los mayores retos para los profesionales de la salud y las instituciones prestadoras de atención en salud porque afecta la calidad de vida de las personas, porque tienen que someterse a un tratamiento de por vida y por las complicaciones que se generan debido a esta enfermedad (5).

La brecha entre el conocimiento y el comportamiento de los pacientes identificados por la literatura no es insuperable, pero debe ser enfrentada por los profesionales de la salud como un desafío que puede ser superado a través de programas educativos. 
La educación ofrece a los pacientes el acceso a herramientas que son útiles para el desarrollo de habilidades de autocuidado y apunta a aumentar su nivel de conocimiento (24). La adquisición de estas habilidades es calificada como una estrategia eficaz para fortalecer su motivación para cumplir con el plan de la dieta, aumentar su actividad física, fortalecer el cuidado de los pies, medir su nivel de glucosa en sangre capilar, prevenir episodios de hipoglucemia y complicaciones crónicas y gestionar su enfermedad bajo circunstancias especiales (24).

En el presente estudio se realizó una caracterización con diversas variables. En cuanto a los factores sociodemográficos, se encontró que la mayoría de los participantes eran mujeres, casadas o solteras y dedicadas al hogar, como se aprecia en otras investigaciones realizadas por Alves (24), Gomes (25), Galhardo (26). El estado civil, y más específicamente la situación de viudez, puede interferir en el manejo de la diabetes, ya que en algunos casos la pérdida del compañero provoca alteraciones de salud como depresión, desánimo y pérdida del deseo de vivir (27).

La edad, el nivel de escolaridad y la ocupación son variables que pueden influir en el control metabólico (28). La edad mínima de aparición, en esta investigación, fue de 30 años, contrario a otros estudios donde se afirma que es de 40 años (29-30), lo cual constituye un dato preocupante y requiere de intervención inmediata por parte de los profesionales de la salud, evitando que la edad de aparición de la enfermedad sea cada vez menor.

La prevalencia de mujeres es alta para el padecimiento de esta enfermedad y se relaciona con los resultados de la investigación realizada por Ruiz (31). Hallazgos similares se encontraron en un estudio colombiano desarrollado por Otero (27), cuya edad en promedio fue de 62 años y el sexo predominante fue el femenino, datos que se relacionan con lo propuesto por la OMS en donde se afirma que las muertes por diabetes corresponden a personas menores de 70 años $\mathrm{y}$, de este porcentaje, un $55 \%$ son mujeres (3).

En los resultados de este estudio también se encontró que la edad media fue de 60 años, el tiempo de diagnóstico de la enfermedad fue mayor a 10 años, casi la mitad de los pacientes se encontraba con diagnóstico de obesidad y aproximadamente la cuarta parte presentó diagnóstico de sobrepeso. Estos resultados son similares al estudio de Echenique (15), desarrollado con 115 pacientes, en donde la edad promedio al ingreso en el programa fue de 62.2 años para los hombres y 60.2 años para las mujeres, el tiempo mediano de diagnóstico de la enfermedad fue 10.5 años, el $46 \%$ de los pacientes presentaba obesidad y el $40,7 \%$ sobrepeso.

Del total de pacientes con diabetes, un gran porcentaje $(86,9 \%)$ fue citado por parte del ortopedista, pero solamente cerca de la mitad de ellos cumplió con el control requerido. Como diagnóstico médico de patología crónica agregada, la mayoría presentó hipertensión arterial. Estos resultados son similares a los obtenidos en un estudio de Silva (32), donde el 98,3 $\%$ de los individuos consideraba importante cuidar de los pies para prevenir el pie diabético, mientras que el $81 \%$ refería no realizar examen de los pies por lo menos una vez al año con el profesional de salud; además, la mayoría de los individuos presentaba hipertensión arterial asociada a diabetes mellitus tipo 2 .

En los resultados de Silva (32) se comprobó que el $63,8 \%$ hacía uso de hipoglucemiante oral, el 12,0\% usaba apenas insulina y el 17,2\% hipoglucemiante oral asociado a la insulina, datos contrarios a los encontrados en este estudio, donde el tratamiento con medicación mixta correspondió en más de la mitad de los casos, más de la cuarta parte fue sólo por vía oral y en un bajo porcentaje con insulina.

En cuanto a los resultados del cumplimiento de citas por parte del médico y la enfermera, este fue del total de la muestra. El control semestral con el nutricionista correspondió a cerca de las tres cuartas partes y del ortopedista -que se debe realizar cada año- fue un poco más de la mitad, similar a lo encontrado en un estudio de Ávila (11), donde se encontró buen porcentaje de cumplimiento de los controles, permitiendo un adecuado seguimiento de los pacientes por parte del programa y, además, una adecuada detección de pacientes con sobrepeso y obesidad, pero no se evidencia el seguimiento ni control para que el paciente llegue a un estado nutricional adecuado. En este estudio de Ávila (11) también se evidenció que el cumplimiento general en valoraciones adicionales fue del $19 \%$, razón por la cual se esperaría que todos los pacientes tuvieran una valoración por cada una de las áreas especializadas.

Con respecto a la hemoglobina glicosilada, el parcial
E-ISSN 2322-7028

Vol. 14 No. 2

Jul - Dic 2017

Cúcuta, Colombia 
ISSN-PRINT

1794-9831

E-ISSN 2322-7028

Vol. 14 No. 2

Jul - Dic 2017

Cúcuta, Colombia de orina y la micro albuminuria se encontró un porcentaje alto de cumplimiento. Estos hallazgos son diferentes a los de la investigación presentada por Ávila (11), donde se encontró que el $39 \%$ de los pacientes estuvieron controlados a través de la hemoglobina glicosilada y que "los laboratorios para detección temprana de insuficiencia renal, como el parcial de orina y la microalbuminuria, no se están solicitando de acuerdo a lo sugerido" (11). En el estudio de Múnera (33) se propone "utilizar la hemoglobina glicosilada como marcador de seguimiento y como método diagnóstico, ya que, en condiciones normales, refleja la concentración de glucosa en sangre en un periodo de 2 a 3 meses anteriores al análisis".

En el presente estudio, el tiempo de diagnóstico fue de más de 10 años, considerando que el tiempo que llevan en el programa es menos de 1 año, lo que implica la aparición de varias complicaciones y un control tardío de las mismas. Según el estudio planteado por Medina (16), para el momento en que el paciente ingresa al programa, el tiempo que llevaba de diagnóstico de la enfermedad es superior a cinco años, periodo durante el cual el paciente ha podido desarrollar complicaciones secundarias a la diabetes, lo cual puede relacionarse con la ausencia de cambios en el estilo de vida, la irregularidad en la toma de la medicación o la falta de control metabólico a pesar de la toma de la misma. Por otra parte, el estudio de Rodrigues (34) reportó una relación inversa con la adherencia al tratamiento, es decir, a mayor tiempo con el diagnóstico más baja la adherencia.

Las complicaciones de la diabetes mellitus tipo 2 más frecuentes fueron: neuropatía, nefropatía y retinopatía diabética, siendo la complicación más prevalente en este estudio la nefropatía diabética, fenómeno diferente a lo encontrado en el estudio de Canciano (35), donde el $94 \%$ de los pacientes presentó complicaciones de órganos diana, predominando la cardiopatía isquémica con $33 \%$. El 26,5 \% de los pacientes del actual estudio presentó neuropatía diabética, mayor a la encontrada en la investigación propuesta por Santos (36), donde la frecuencia fue de un $14,5 \%$; la frecuencia de retinopatía diabética en este estudio fue $20 \%$ menor a la encontrada en el estudio de Canciano (35), que mostro una ocurrencia de $42,8 \%$.

La alta prevalencia de estas complicaciones afecta la calidad de vida de muchos pacientes y, así mismo, la adherencia terapéutica. $\mathrm{Al}$ respecto se ha descrito que del $20 \%$ al $25 \%$ de los diabéticos tipo 2 tienen riesgo de evolucionar a la nefropatía diabética (37). La neuropatía periférica es una complicación frecuente; dos de cada tres diabéticos la pueden presentar y es el principal factor de riesgo para desarrollar una úlcera, que es la antesala de una amputación (38). La retinopatía diabética representa el 4,8\% de las causas de ceguera a nivel mundial (39). La diabetes es la causa principal de enfermedad renal terminal con la necesidad de hemodiálisis (40).

En la mayoría de historias clínicas analizadas no se encuentra registrado el riesgo de pie diabético; sin embargo, un alto porcentaje no presentó ningún riesgo de pie diabético, similar a lo reportado por Ávila (11), en donde los registros por especialistas y profesionales con segunda especialidad fueron los que más fallas presentaron. Al respecto, se considera que la identificación de la neuropatía diabética y de la enfermedad arterial periférica, a través de la anamnesis y examen físico, son de primera línea para la identificación de estas complicaciones con consecuencias devastadoras, que en la mayoría de los casos se puede prevenir (41) con la aplicación de escalas como las de Meggitt-Wagner, Gibbons y Brodsky, entre otras (42).

La detección e identificación de los factores de riesgo asociados al pie diabético deben ser una prioridad para el profesional de enfermería que trabaje con pacientes diabéticos. Esta detección se realiza en el ámbito de atención primaria mediante métodos exploratorios sencillos y con un coste aceptable (33).

Las complicaciones de la enfermedad, antes mencionadas, se relacionan con el estado de descompensación de la enfermedad que presentaron la mayoría de los pacientes, representado por valores de hemoglobina altos. Así mismo, en otras investigaciones (43-44) se reportaron valores de hemoglobina glicosilada entre $7 \%$ y $8 \%$, lo que se traduce en una situación alarmante en esta población, debido a que la hemoglobina glicosilada se considera un indicador importante del control glicémico de una persona con diabetes en los últimos tres meses.

Uno de los diagnósticos médicos de patologías crónicas que fue común en estos resultados, en casi la cuarta parte de la población, fue el hipotiroidismo, 
mientras que en otro estudio (45) el nivel de hormona estimulante de la tiroides (TSH) en suero fue mayor en los pacientes con diabetes mellitus tipo 2 que en los pacientes con pre-diabetes. Además, la presencia de dislipidemia se asocia, según el estudio de Lahsen (46), con la consecuencia más grave de la lipotoxicidad que se manifiesta en las células beta, ya que la excesiva acumulación de triglicéridos en los islotes pancreáticos aumenta la expresión de la enzima óxido nítrico sintetasa inducible, incrementando los niveles de óxido nítrico y produciendo alteración en la función y, finalmente, apoptosis beta celular.

La gran cantidad de anomalías lipídicas en los diabéticos tipo 2 requiere la normalización del perfil de lípidos para un adecuado control de la dislipemia diabética, ya que los niveles elevados de triglicéridos son fuertes predictores de enfermedad coronaria (EC) en estos pacientes (47). La determinación de micro albuminuria es un importante predictor de riesgo cardiovascular y renal (48). En general, "los pacientes diabéticos tienen mayor incidencia de enfermedad coronaria arteriosclerótica; además los vasos muestran mayor grado de afectación, con lesiones de mayor severidad y extensión" (49). De otro lado, la retinopatía diabética es asintomática y se detecta por un fondo de ojo, razón por la cual es esencial un examen anual de la retina (50).

Al respecto, teóricamente se considera que en la población de pacientes diabéticos la prevalencia de hipertensión es 1,5 a 3 veces mayor que en los no diabéticos. En la diabetes, la hipertensión puede ya estar presente en el momento del diagnóstico o inclusive puede preceder a la hiperglicemia evidente (51) y presenta asociación significativa con las dislipidemias y con el antecedente familiar de diabetes (52).

El registro del diagnóstico nutricional se ve evidenciado por un alto porcentaje de obesidad y sobrepeso, similar a los resultados del trabajo de Zubizarreta (53), en donde se encontró un 48,3\% de pacientes obesos, un 32,5\% con sobrepeso y un $19,2 \%$ con normo peso, resultados que avalan que la prevalencia de la diabetes va ligada a la obesidad.

Los datos de las historias clínicas mostraron cumplimiento en la aplicación de los laboratorios correspondientes para el adecuado diagnóstico, al realizar la comparación con lo planteado en la guía de práctica clínica sobre diabetes tipo 2 (54), donde la glicemia y la hemoglobina glicosilada se realizan cada tres meses y el perfil lipídico, parcial de orina, microalbuminuria y la creatinina se realizan cada año (54), excepto el control con el electrocardiograma, que se debe hacer anualmente y, en este caso, se realizó a más de la tercera parte de los pacientes. El examen de fondo de ojo se realizó a una gran parte de los pacientes, según la Guía de práctica clínica para el diagnóstico, tratamiento y seguimiento de la DM Tipo 2 del 2015 (55), que refiere que se debe realizar al menos una vez por año y remitir al oftalmólogo al momento del diagnóstico en DM tipo 2 y, luego, cada dos años.

La importancia de la realización de este examen radica en la posibilidad de identificar el daño cerebrovascular, riesgo de accidente cerebrovascular (ACV), enfermedad coronaria, insuficiencia cardíaca y morbimortalidad cardiovascular, que en muchos casos se suma al diagnóstico de diabetes (56).

El tratamiento más utilizado para el control de la diabetes tipo 2 fue el mixto; sin embargo, el trabajo de González (43) refiere que se dosifica más el tratamiento con hipoglicemiantes orales. El estudio de Sanz (57), por su parte, mostró que antes de la admisión a hospitalización de los pacientes con DM tipo 2, el 33,3\% se encontraba con insulina y el 69,1 $\%$ con hipoglucemiantes orales. Independientemente del tratamiento, es importante que éste se ajuste a las necesidades personales de los pacientes, siendo de vital importancia para su cumplimiento la sensibilización de los pacientes para evitar el abandono de la medicación prescrita y el conocimiento sobre los efectos y signos de alarma para que sean notificados oportunamente a los especialistas. Esto coincide con lo presentado en la investigación de Delgado (58), quien afirma que las personas con diabetes necesitan revisar y actualizar su conocimiento, ya que constantemente se están desarrollando nuevas investigaciones y mejores maneras de tratar la enfermedad. El diabético tiene que realizar un laborioso autocuidado, que va desde el autoanálisis y el ajuste del tratamiento dietético y farmacológico, además del tratamiento de técnicas de autoinyección, hasta otros aspectos como el cuidado de los pies, la higiene y el ejercicio físico.

Ramos et al. (59) refieren que "la mayoría de las guías recomienda utilizar insulina ante un paciente mal controlado con 2 antidiabéticos orales", puesto que 
ISSN-PRINT

1794-9831

E-ISSN 2322-7028

Vol. 14 No. 2

Jul - Dic 2017

Cúcuta, Colombia la producción y "reserva de insulina se han reducido a un nivel crítico y la célula beta no responde al estímulo de los fármacos con acción secretagoga".

En el presente estudio, el hipoglicemiante ordenado a los pacientes con mayor frecuencia fue la metformina. Al respecto, Salazar (60) menciona que "numerosos estudios han demostrado que su uso asociado a medidas que cambian el estilo de vida, puede disminuir la proporción de personas con intolerancia a los glúcidos que de otra manera evolucionarían a una diabetes".

Otros medicamentos de uso frecuente son la glibenclamida, glimepirida y metformina, que tienen limitado su uso en la mayoría de las complicaciones vasculares de la diabetes, por lo cual se realiza la administración de insulina (59), mientras que otros pacientes tienen ordenado vildagliptina o sitagliptina con metformina. Este tratamiento induce a una disminución de la respuesta postprandial de triglicéridos en un $70 \%$ de los pacientes, acompañado de un mejor control de la glucosa y mejorando el metabolismo glucídico y el estado lipemico postpandrial (61).

El control por parte del profesional de enfermería y el médico fue alto comparado con el de los profesionales de nutrición y ortopedia. En este sentido, es recomendable la vigilancia y el estricto cumplimiento de controles médicos de los pacientes, las interconsultas especializadas según requerimientos y la adecuada valoración integral del diabético (62).

En relación con el control por ortopedista, un poco más de la mitad no tiene el registro por parte del especialista; la gran mayoría de los pacientes fueron citados, pero sólo alrededor de la mitad asistieron al control. Esta situación conlleva a la necesidad de verificar la información de manera permanente, no solamente con el registro en las historias clínicas sino también con el seguimiento a los pacientes cuando no asisten, ya que se ha evidenciado, en el estudio propuesto por Ramos (63), que a mayor control, menor probabilidad de amputación de extremidades.

En cuanto al régimen alimenticio indicado en el último control, a más de la mitad de los pacientes se les brindó educación, es decir, se le dieron instrucciones; sin embargo, las altas estadísticas de obesidad y sobrepeso no concuerdan con el autocuidado del paciente. En la investigación de Silva (32) se muestra claramente que, en los hábitos comportamentales, el $56,1 \%$ de los pacientes no seguía las orientaciones nutricionales; del mismo modo, el 53,7 \% no practicaba ejercicio físico y el $14,6 \%$ era tabaquista. El control de los factores de riesgo a los que están expuestos los pacientes del programa de diabetes se logró amortiguar, por parte de enfermería, a través del desarrollo de talleres educativos, donde se abordaron temas como estilos de vida saludables, complicaciones crónicas de la diabetes, insulinas y automonitoreo, adicional a la citación de los pacientes mensualmente para realizar toma de signos vitales, peso, talla, índice de masa corporal y medición de perímetro abdominal.

Vale la pena destacar la labor que viene desarrollando el equipo interdisciplinario, puesto que se maneja un alto volumen de pacientes. Estudios al respecto (64) (16) destacan la importancia del control médico y de enfermería porque se mejora la respuesta metabólica y la calidad de vida de los pacientes. En ese sentido, se considera que el seguimiento de los pacientes mejora los resultados del proceso (18), en especial cuando se desarrolla un trabajo multidisciplinar con abordaje integral (65).

En el trabajo de Echenique (15) se afirma que un programa multidisciplinario para diabéticos, bien estructurado, mejora las metas metabólicas de los pacientes con diabetes mellitus tipo 2. Para Barrera (66), el objetivo de la educación debe centrarse en proporcionar al paciente la información necesaria para que se responsabilice de su autocuidado y, de esta manera, prevenga el desarrollo de complicaciones asociadas.

Para Caicedo et al. (4), "el control del tratamiento de la diabetes mellitus tipo 2 depende de múltiples factores, es de vital importancia para evitar las complicaciones de los pacientes y no sólo abarca un tratamiento farmacológico sino también una serie de modificaciones en el estilo de vida, como la adherencia al tratamiento"

De acuerdo con la revisión bibliográfica, los resultados de la presente investigación y la experiencia con pacientes crónicos, en especial con diabéticos, es posible afirmar que son susceptibles de generar complicaciones graves que limitan su productividad y años de vida perdidos. Esto, a su vez, afecta la economía del hogar, produce inestabilidad 
familiar, mayor demanda de la atención y costos por parte de las instituciones de salud. Por lo tanto, se requiere de programas bien estructurados, con personal interdisciplinario competente, que permitan identificar las necesidades individuales y redes de apoyo para la consecución de los cuidados y el cumplimiento del régimen terapéutico prescrito.

El liderazgo de los profesionales de la salud y el compromiso con los pacientes contribuye al mejoramiento de su calidad de vida, ya que es muy triste encontrar a pacientes con deterioro y en muchos de los casos con expresiones como "si me hubieran explicado no me hubiera quedado ciego" y "ya qué se puede hacer, me tiene que quitar ese pie" (Individuo No. 32).

\section{Conclusiones}

El grupo de estudio, en su mayoría, es de sexo femenino, se dedica al hogar y se encuentra en edades comprendidas entre los 50 y los 59 años. Llevan en su mayoría más de 10 años con la enfermedad, el valor de la $\mathrm{HbA} 1 \mathrm{c}$ se encuentra normal únicamente en el 21 $\%$ de los pacientes, el 80,9\% presenta hipertensión arterial y el 72,6 \% nefropatía diabética como complicación de la enfermedad. Se resalta el alto riesgo de enfermedades cardiovasculares reflejado en los pacientes que se encuentran con diagnóstico de obesidad, equivalente al $46,9 \%$.

El tipo de atención otorgada es considerada de ámbito interdisciplinario -en donde participan todos los profesionales de la salud- y el cumplimiento de los pacientes a los controles programados es bastante favorable, evidenciado en el $100 \%$ de cumplimento de citas al mes por parte del médico y la enfermera y un $71,7 \%$ por parte de la nutricionista, semestralmente.

Los pacientes, a pesar de estar en un programa de atención integral de diabetes mellitus tipo 2, aún no tienen un óptimo control glicémico, lo cual sugiere la revisión del cumplimiento del régimen terapéutico por parte de ellos, para prevenir complicaciones que se puedan presentar a corto, mediano o largo plazo.

Es importante el registro, en las historias clínicas, de lo relacionado con el riesgo de pie diabético, ya que permite realizar un análisis de confiabilidad alta y proponer intervenciones que respondan a los hallazgos encontrados.

Se observó alta adherencia a los talleres educativos presentados por la enfermera sobre estilos de vida saludables, complicaciones crónicas de la diabetes, insulinas y automonitoreo. Esto refleja la valiosa labor del profesional de enfermería en el ámbito de educación.

\section{Conflicto de intereses}

Las autoras declaran no tener ningún conflicto de intereses.

\section{Referencias bibliográficas}

1. Casanova M, Delgado M, Odalis O, Pratz OM, Gomez DB. Nivel de conocimientos sobre la enfermedad en los adultos mayores con diabetes mellitus tipo 2. Rev Ciencias Médicas 2011; 15(2): 122-132.

2. Almaguer A, Miguel PE, Soca M, Será CR, Mariño A, Oliveros R. Actualización sobre diabetes mellitus. CCM 2012; 16(2).

3. Jansà M, Vidal M. Importancia del cumplimiento terapéutico en la diabetes mellitus. Av Diabetol. 2009; 25(1): 55-61.

4. Caicedo DC, Duarte CA, González A, Gualdrón EL, Gualdrón $R L$, Igua DM, et al. Factores no farmacológicos asociados al control de la diabetes mellitus tipo 2. MÉD.UIS. 2012; 25(1): 29-43.

5. López-Ramón C, Ávalos-García MI. Diabetes Mellitus hacia una perspectiva social. Revista Cubana de Salud Pública [Internet]. 2013 [consultado 16 de enero de 2015]; 39(2). Disponible en: http://bvs. sld.cu/revistas/spu/vol39_2_13/spu13213.htm

6. Sánchez-Herrera B, Carrillo-González GM, Barrera-Ortiz L, Chaparro-Díaz L. Carga del cuidado de la enfermedad crónica no transmisible. Aquichan. 2013; 13(2): 247-60.

7. Preciado DC, Tovar JR, Correa JE. Costo-efectividad de un programa de actividad física, dirigida en un departamento de Colombia. Investig Andin. 2012; 14(25): 502-15. 
ISSN-PRINT

$1794-9831$

E-ISSN 2322-7028

Vol. 14 No. 2

Jul - Dic 2017

Cúcuta, Colombia

8. Villarreal E, Romero K, Vargas E, Galicia L, Martinez G, Ramos J. Coste-efectividad del uso de esquemas de tratamiento farmacológico en pacientes con diagnóstico de diabetes mellitus tipo 2 . Rev Clínica Med Fam. 2012; 5(3): 158-65.

9. Ledón-Llanes L. Impacto psicosocial de la diabetes mellitus, experiencias, significados y respuestas a la enfermedad. Rev Cubana Endocrinol [Internet]. 2012 [consultado 20 de abrilde 2015]; 23(1). Disponible en: http://scielo.sld.cu/scielo.php?script=sci_arttext\&pid=S1561-29532012000100007\&lng=es

10. Contreras-Orozco A, Contreras-Machado A, Hernández-Bohórquez C, Castro Balmaceda M, Navarro Palmett L. Capacidad de autocuidado en pacientes diabéticos que asisten a consulta externa. Sincelejo, Colombia. Self-care Capacit [Internet]. 2013 [consultado 3 febrero de 2015]; 15(26). Disponible en: http:// search.ebscohost.com/login.aspx?direct=true\&db=fua\&AN= 86950640\&lang=es\&site=ehost-live

11. Ávila JC, Bareño A, Castro J, Rojas C. Evaluación de la aplicación de las guías de hipertensión y diabetes en un programa de crónicos. rev. fac. med 2014; 22(2): 58-67.

12. Arnold-Rodríguez $M$, Arnold-Domínguez $Y$, Alfons-Hernández $Y$, Villar-Guerra $C$, González-Calero TM. Pesquisaje y prevención de la diabetes mellitus tipo 2 en población de riesgo Screening and prevention of diabetes mellitus type 2 in risky population. Rev Cubana Hig Epidemiol. 2012; 50(3): 380-91.

13. Osuna M, Rivera MC, Bocanegra CJ, Lancheros A, Tovar H, Hernández JI, et al. Caracterización de la diabetes mellitus tipo 2 y el control metabólico en el paciente hospitalizado. Acta Médica Colombiana 2014; 39(4): 344- 351.

14. Gómez DA, Mahecha M, Gómez RD, Agudelo SM. Características de la Diabetes Mellitus en una red de servicios de primer de nivel de atención. Medellín, 2005-2008. Rev. Fac. Nac. Salud Pública 2011; 29(1): 7-17.

15. Echenique-Sarah J, Rodríguez-Osiac L, Pizarro-Quevedo T, Martín MA, Atalah Samur E. Impacto de un programa nacional de tratamiento en mujeres adultas con exceso de peso en centros de atención primaria. Nutr Hosp. 2011; 26(6): 1372-7.

16. Medina A, Ellis E, Ocampo D. Impacto de un programa personalizado de educación en pacientes diabéticos tipo 2. Acta Médica Colomb 2014; 39(3): 258-63.

17. Cáceres-Martínez L, Aliaga-Llerena K, Bendezú-Loredo Z, Bendezú-Carrasco E, Monja-Yturregui J. Evaluación del conocimiento sobre Diabetes Mellitus en pacientes diabéticos tipo 2 . Revista de la Facultad de Medicina Humana [Internet]. 2007 [consultado 29 de julio de 2015]; 7(2). Disponible en: http://aulavirtual1.urp.edu.pe/ojs/index.php/RFMH/article/view/100

18. Galiano M, Calvo M, Feito M, Aliaga M, Leiva S, Mujica B. Condición de salud y cumplimiento de la guia clinica chilena para el cuidado del paciente diabetico tipo 2. Rev. Cienc. enferm [Internet]. 2012 [consultado 11 de mayo de 2016]; 18 (3). Disponible en: http://www.scielo.cl/scielo. php?script=sci_arttext\&pid=S0717-95532012000300006\&lng=es. http://dx.doi.org/10.4067/S071795532012000300006.

19. República de Colombia, Ministerio de Salud. Resolución No 008430 de 1993, octubre 4, por la cual se establecen las normas científicas, técnicas y administrativas para la investigación en salud. [Internet]. Santa Fé de Bogotá: El Ministerio; 1993 [consultado 29 de enero de 2015]. Disponible en: http://www.unisabana. edu.co/fileadmin/Docu mentos/Investigación/comite_de_etica/Res_8430_1993___Salud.pdf

20. Serrano- LaVertu D, Linares- Parada, A. Principios éticos de la investigación biomédica en seres humanos: aplicación y limitaciones en américa latina y el caribe. Bol of Sanit Panam [Internet]. 1990 [consultado 10 de agosto de 2015]; 108(5-6): 489-499. Disponible en: http://iris.paho.org/xmlui/ bitstream/handle/123456789/16826/v108n\%285-6\%29p489.pdf?sequence=1\&isAllowed=y

21. González-Tabares R, Aldama-Leonard IY, Fernández-Martínez L, Ponce-Baños I, Rivero Hernández MC, Jorin-Castillo N. Hemoglobina glucosilada para el diagnóstico de diabetes mellitus en exámenes médicos preventivos. Rev Cuba Med Mil [Internet]. 2015 [consultado 29 de marzo de 2015]; 44(1). Disponible en: http://www.bvs.sld.cu/revistas/mil/vol44_1_15/mil07115.htm

22. Pereira- Despaigne O, Palay- Despaigne M, Rodríguez- Cascaret A, Neyra- Barros, R, Chia Mena M. Hemoglobina glucosilada en pacientes con diabetes mellitus. MEDISAN [Internet]. 2015 [consultado 
30 de septiembre de 2015]; 19(4). Disponible en: http://scielo.sld.cu/scielo.php?script=sci arttext\&pid=S1029-30192015000400012\&lng=es

23. León-Mazón M, Araujo-Mendoza G, Linos-Vázquez Z. Eficacia del programa de educación en diabetes en los parámetros clínicos y bioquímicos. Rev Med Inst Mex Seguro Soc. [Internet]. 2012 [consultado 7 de abril de 2015]; 51(1). Disponible en: http://www.medigraphic.com/pdfs/imss/im2013/im131j.pdf

24. Alves- Chagas I, Josana C, Dos- Santos M, Luchetti- Rodrigues F, Alves- Arrelias C, Teixeira- de Souza C, et al. Conhecimento de pacientes com diabetes sobre tratamento após cinco anos do término de um programa educativo. Rev Esc Enferm USP 2013; 47(5): 1141-6.

25. Gomes-Villas Boas LC 1 , Foss MC, Freitas MC, Pace AE. Relationship among social support, treatment adherence and metabolic control of diabetes mellitus patients. Rev Lat Am Enfermagem [Internet]. 2012 [consultado 15 de marzo de 2015]; 20(1). Disponible en: http://www.ncbi.nlm. nih.gov/pubmed/24553698\nhttp://www.scielo.br/scielo.php?script=sci_arttext\&pid=S0104$11692014000100011 \& \operatorname{lng}=$ en\&nrm=iso\&tlng=en $\backslash n w w w . e e r p . u s p . b r / r l a e$

26. Galhardo AL, Gomes LC, Foss de Freitas MC, Foss MC, Pace AE. Perception of social support by individuals with diabetes mellitus and foot ulcers . Acta Paul Enferm. 2012; 25(Esp 1): 20-6.

27. Otero LM, Zanetti ML, Teixeira CR. Sociodemographic and clinical characteristics of a diabetic population at a primary level health care center. Rev Lat Am Enferm [Internet]. 2007 [consultado 4 de febrero de 2015]; 15 (Spec): 768-73. Disponible en: http://www.ncbi.nlm.nih.gov/pubmed/17934583

28. Solano- Solano G, Pace A, García- Reza C, Arreda- Del Castillo A. Efectos de un protocolo aplicado por enfermeras en el control metabólico a personas con diabetes tipo 2. Cienc. enferm [Internet]. 2013 [consultado 09 de junio de 2015]; XIX (1). Disponible en: http://www.scielo.cl/scielo. php?script=sci_arttext\&pid=S0717-95532013000100008\&lng=es http://dx.doi.org/10.4067/S071795532013000100008

29. Regueira- Betancourt S, Díaz- Perez M, Cajape- Leon L, Jiménez- Batioja G. Farmacoterapéutica en pacientes diabéticos de un consultorio médico de la familia. Revista Electrónica Dr. Zoilo E. Marinello Vidaurreta [Internet]. 2016 [consultado 1 de febrero de 2016]; 41(6). Disponible en: http:// revzoilomarinello.sld.cu/index.php/zmv/article/view/755

30. Lovera MN, Castillo MS, Malarczuk C, Castro C, Bonneau GA, Ceballos BH, et al. Incidencia de Diabetes Mellitus tipo 2 y factores de riesgo en una cohorte de trabajadores de la salud. Acta Bioquim Clin Latinoamerica. 2014; 48(1): 45-52.

31. Ruiz D, García A, Antunez J, Rodríguez L. Intervención educativa sobre Diabetes Mellitus en pacientes portadorea de la enfermedad. AMC. 2011; 15(1): 1-8.

32. Silva L, Rezende M, Ferreira L, Dias F, Helmo F, Silveira F. Cuidados com os pés : o conhecimento de indivíduos com diabetes mellitus cadastrados no programa saúde da familia Enfermería Global 2015; 14 (1): 38-51.

33. Múnera-Jaramillo MI, Restrepo-Lozada MA, Gómez-Bahamón LM, Mesa-Suarez DR, RamirezPuerta BS. Hemoglobina glicosilada A1c vs. glucemia plasmática en ayunas de pacientes ambulatorios de un laboratorio médico. Rev. salud pública. 2011; 13(6): 980-9.

34. Rodrigues F, Dos Santos M, Teixeira C, Gonela Jefferson Thiago, Zanetti Maria Lucia. Relação entre conhecimento, atitude, escolaridade e tempo de doença em indivíduos com diabetes mellitus. Acta paul. enferm. 2012; 25(2): 284-90.

35. Canciano-Chirino E, Reinoso-Pérez M, Martínez-Martínez A, Valdés-Busquet S, Meneses-Rodríguez F. Diabetes mellitus en ancianos, caracterización epidemiológica y comportamiento clínico terapéutico en San Antonio de los Baños. Rev haban cienc méd [Internet]. 2011 [consultado 8 de enero de 2015]; 10(1). Disponible en: http://scielo.sld.cu/scielo.php?script=sci_arttext\&pid=S1729519X2011000100009

36. Santos L, Cecilio HP, Teston EF, de Arruda GO, Peternella FM, Marcon SS. Microvascular complications in type 2 diabetes and associated factors: a telephone survey of self-reported morbidity. Cien Saude Colet [Internet]. 2015; [consultado 11 de enero de 2015]; 20(3). Disponible en: http:// 
ISSN-PRINT

$1794-9831$

E-ISSN 2322-7028

Vol. 14 No. 2

Jul - Dic 2017

Cúcuta, Colombia www.ncbi.nlm.nih.gov/pubmed/25760116

37. Abascal R, Fuentes O, Gutiérrez O, Garay R, Águila O. Nefropatía diabética en pacientes diabéticos tipo 2. Rev med. 2011; 50(1): 29-39.

38. Ibarra C, Rocha J, Hernández R, Nieves R , Leyva R. Prevalencia de neuropatía periférica en diabéticos tipo 2 en el primer nivel de atención. Rev Med Chil [Internet]. 2012 [consultado 12 de enero de 2015]; 140(9). Disponible en: http://www.scielo.cl/scielo.php?script=sci_arttext\&pid=S003498872012000900004\&lng=es\&nrm=iso\&tlng=es

39. Castro- Cardenas K, López- Dorta N, Rodríguez- Rivero D, Suárez- Pérez J, Llerena- Rodríguez J. Factores de riesgo y severidad de la retinopatía diabética. Rev Cub Med Mil [Internet]. 2013 [consultado 6 de mayo de 2015]; 42(2). Disponible en: http://www.bvs.sld.cu/revistas/mil/vol42_2_13/mil07213.htm

40. Burgos-Peláez R, Joaquim C, Puiggrós-Llop C, Chicharro-Serrano LL. Diabetes mellitus tipo 2 crónica. Nutr Hosp Supl. 2010; 3(1): 35-45.

41. Pinilla AE, Barrera MP, Sánchez AL, Mejía A. Factores de riesgo en diabetes mellitus y pie diabético: un enfoque hacia la prevención primaria. Rev. Colomb. Cardiol. [Internet]. 2013 [consultado 14 de enero de 2015]; 20(4). Disponible en: http://www.scielo.org.co/scielo.php?script=sci_arttext\&pid $=\mathrm{S} 0120-56332013000400008$.

42. González H, Mosquera A, Quintana M, Perdomo E, Quintana M. Clasificaciones de lesiones en pie diabético. Un problema no resuelto. Gerokomos. 2012; 23(2): 75-87.

43. González-Tabares R, Aldama-Leonard IY, Fernández-Martínez L, Ponce-Baños I, Rivero Hernández MC, Jorin-Castillo N. Hemoglobina glucosilada para el diagnóstico de diabetes mellitus en exámenes médicos preventivos. Rev Cuba Med Mil [Internet]. 2015 [consultado 29 de marzo de 2015]; 44(1). Disponible en: http://www.bvs.sld.cu/revistas/mil/vol44_1_15/mil07115.htm

44. Lenz R, Zarate A, Rodriguez J, Ramirez J. Niveles de hemoglobina glicosilada y diferencia en el gasto en salud de pacientes diabéticos: un estudio econométrico. Rev Med Chil. 2014; 142: 841-9.

45. Duran AO, Anil C, Gursoy A, Nar A, Inanc M, Bozkurt O, et al. Thyroid volume in patients with glucose metabolism disorders. Arq Bras Endocrinol Metab. 2014; 58(8): 824-7.

46. Lahsen R. Síndrome metabólico y diabetes. REV. MED. CLIN. CONDES [Internet]. 2014 [consultado 29 de enero de 2015]; 25(1): 47-52. Disponible en: http://www.sciencedirect.com/science/article/pii/ S0716864014700100

47. Arruzazabala ML, López E, Molina V, 1llnait J, Carbajal D, Mas R, et al. Efectos del D-003 sobre el perfil lipídico y la agregación plaquetaria en pacientes con diabetes tipo 2. Acta Bioquím Clín Latinoam 2010; 44(1): 15-24.

48. Lou-Arnal LM, Campos-Gutiérrez B, Cuberes-Izquierdo M, Gracia-García O, Turón-Alcaine JM, Bielsa-García S, et al. Prevalence of chronic kidney disease in patients with type 2 diabetes mellitus treated in primary care. Nefrologia [Internet]. 2010 [consultado 29 de enero de 2015]; 30(5). Disponible en: http://www.ncbi.nlm.nih.gov/pubmed/20882094

49. Piña-Rivera Y, Cruz- Hernández L, Parlá-Sardiñas Y, Fernández- Marrero M. Isquemia miocárdica silente en diabéticos tipo 2. Rev Cuba Endocrinol [Internet]. 2012 [consultado 14 de enero de 2015]; 23(2). Disponible en: http://scielo.sld.cu/scielo.php?script=sci_arttext\&pid=S1561-29532012000200004

50. Rodríguez-Rodríguez B. Prevención de ceguera por retinopatía diabética : ¿dónde estamos? Rev Cuba Oftalmol. 2015; 28(1): 119-28.

51. Sánchez R, Ayala M, Baglivo H, Velázquez C, Burlando G, Kohlmann O, et al. Guías Latinoamericanas de Hipertensión Arterial. Rev Chil Cardiol [Internet]. 2010 [consultado 13 de abril de 2015]; 29(1). Disponible en: http://www.scielo.cl/pdf/rchcardiol/v29n1/art12.pdf

52. Cardona J, Rivera Y, Llanes O. Prevalencia de diabetes mellitus y dislipidemias en indígenas del resguardo Cañamomo-Lomaprieta, Colombia. Investig. andina 2012; 14(24): 414-26.

53. Zubizarreta K, Rodríguez N, Castilla A, Costa M, Becerra N. Relación entre características antropométricas y su repercusión en diabéticos tipo 2. Rev Ciencias Médicas 2012; 16(2): 3-19.

54. Oviedo MA, Espinosa F, Reyes H, Pérez JA, Gil E. Guía clínica para el diagnóstico y tratamiento de la diabetes mellitus tipo 2. Rev Med IMSS [Internet]. 2004; [consultado 17 de mayo de 2015] 41(Supl): 
S27-S46. Disponible en: http://www.medigraphic.com/pdfs/imss/im-2003/ims031d.pdf

55. República de Colombia, Ministerio de Salud y Protección Social. Guía de práctica clínica para el diagnóstico, tratamiento y seguimiento de la DM Tipo 2 en la población mayor de 18 años. [Internet]. 2015 [consultado el 18 de abril del 2016]. Disponible en: http://gpc.minsalud.gov.co/gpc_ sites/Repositorio/Conv_637/GPC_diabetes/GPC_diabetes_tipo2_completa.aspx

56. Sociedad Argentina de Cardiología. Consenso de diagnóstico, prevención y tratamiento de la cardiotoxicidad por tratamiento médico del cáncer. Revista Argentina de Cardiología [Internet]. 2013 [consultado 12 de febrero de 2015]; 81(Suppl 5): 1-60. Disponible en: http://educacion.sac.org. ar/pluginfile.php/12892/mod_resource/content/1/Consenso-Cardiotoxicidad\%20lite.pdf.

57. Sanz-París A, García JM, Gómez-Candela C, Burgos R, Martín Á, Matía P, et al. Malnutrition prevalence in hospitalized elderly diabetic patients. Nutr Hosp [Internet]. 2013; [consultado 5 de junio de 2015] 28(3). Disponible en: http://www.ncbi.nlm.nih.gov/pubmed/23848076

58. Pérez- Delgado A, Alonso- Carbonell L, García- Milián A, Garrote- Rodríguez I, González- Pérez S, Morales- Rigau J. Intervención educativa en diabéticos tipo 2. Rev Cubana Med Gen Integr [Internet]. 2009 [consultado el 03 de diciembre del 2015]; 25(4). Disponible en: http://scielo.sld.cu/scielo. php?script $=$ sci_arttext\&pid=S0864-21252009000400003\&lng=es

59. Ramos E, Camps M, Escalona F. Frecuencia y factores asociados al tratamiento con insulina en diabéticos tipo 2 de la provincia Granma. Rev Cubana Med. 2014; 53(2): 116-26.

60. Salazar-Álvarez Y. Uso de la metformina en la diabetes mellitus tipo II. Rev Cuba Farm. 2011; 45(1): 157-66.

61. Vergel MA, Sala A, Buela L, Valeri L, Arata-Bellabarba G, Velázquez EM. Efecto de la combinación fija de Vildagliptina/Metformina o Sitagliptina/Metformina sobre la lipemia postprandial en pacientes con diabetes tipo 2. Rev. Venez. Endocrinol. Metab. 2012; 10(3): 162-169.

62. González- Rodríguez R, Cardentey- Garcia J, Casanova- Moreno M. Intervención sobre educación nutricional en pacientes con diabetes mellitus tipo 2. AMC [Internet]. 2015 [consultado 29 de octubre de 2015]; 19(3). Disponible en: http://scielo.sld.cu/scielo.php?script=sci_arttext\&pid $=$ S1025-02552015000300008

63. Ramos C, Freese d E, Vieira W, Cavalcanti E. Factors associated with diabetic foot amputations. $\mathrm{J}$ Vasc Bras [Internet]. 2015 [consultado 22 de junio de 2015]; 14(1). Disponible en: http://www.scielo. br/scielo.php?script=sci_arttext\&pid=S1677-54492015000100037.

64. Ochoa-Ortega M, Díaz-Domínguez M, Arteaga-Prado Y, Morejón-Rosales D, Arencibia-Díaz L. Caracterización sociodemográfica y temporal de la diabetes mellitus tipo 2. Rev Ciencias Médicas Pinar del Río [Internet]. 2012 [consultado 17 de enero de 2015]; 16(6). Disponible en: http://scielo.sld. $\mathrm{cu} /$ scielo.php?script=sci_arttext\&pid=S1561-31942012000600006\&lng=es\&nrm=iso\&tlng=es

65. González H, Perdomo E, Quintana M, Quintana M, Mosquera A. Estratificación de riesgo en pie diabético. Gerokomos. 2010; 21(4): 172-82.

66. Barrera P, Pinilla A, Caicedo L, Castillo Y, Lozano Y, Rodríguez K. Factores de Riesgo Alimentarios y Nutricionales en adultos con Diabetes Mellitus. Rev Fac Med Univ Nac Colomb. 2012; 60(1): 28-40. 